Article original

\title{
Evaluation des changements physico-chimiques caractérisant le mûrissement au cours de l'entreposage de trois variétés de bananes Musa spp. (AAB, cv. Corne 1 ; AAA, cv. Poyo et AA, cv. Figue Sucrée)
}

\author{
Kouamé R. N'GanzouA * Brahima Camara \& Emmanuel Dıck
}

Laboratoire de Physiologie Végétale, UFR Biosciences, Université de Cocody - Abidjan, 22 BP 582 Abidjan 22 Côte d'lvoire *Auteur pour les correspondances (E-mail : renenganz@yahoo.fr)

\section{Résumé}

Les propriétés physico-chimiques caractérisant le mûrissement d'une variété de banane plantain (Corne 1) et de deux variétés de bananes de dessert (Poyo et Figue Sucrée) ont été étudiées à la température ambiante $\left(28 \pm 2^{\circ} \mathrm{C}\right)$ et comparées. Les résultats montrent que le délai d'apparition des stades du mûrissement est fonction des variétés, et que le plantain avec un délai de 9 jours mûrit plus lentement que les bananes dessert ( 6 à 7 jours). L'indice de coloration, la résistance pénétrométrique, la teneur en glucides solubles et réducteurs ainsi que l'acidité titrable varient en fonction du stade de mûrissement et des variétés. Les variétés accumulant le plus et le moins de sucres solubles et réducteurs sont la Figue Sucrée $(33,80 \mathrm{mg}$ de glucose/ G. MF) et le plantain (14,52 mg de glucose/ G. MF), le Poyo est intermédiaire (23,03 mg de glucose/ G. MF).

Mots clés : mûrissement, banane, variétés, propriétés physico-chimiques, Musa spp.

\section{Abstract}

Evaluation of the physico-chemical changes characterizing ripening during the storage of three varieties of bananas Musa spp. (AAB, cv. Corn 1; AAA, cv. Poyo and AA, cv. Figue Sucrée)

Physico-chemical properties characterizing the ripening of a variety of plantain (Corn 1) and two varieties of bananas (Poyo and Figue sucrée) were studied at the ambient temperature $\left(28 \pm 2^{\circ} \mathrm{C}\right)$ and were compared. Results show that the time of ripening stages appearance is a function of the varieties, and plantain with 9 days ripen more slowly than bananas ( 6 to 7 days). The index of colouring, the penetrometric resistance, the soluble glucides content and reducing as well as titratable acidity vary according to the stage of ripening and the varieties. The varieties accumulating more and less soluble and reducing sugars are the Figue sucrée (33,80 Mg of glucose/G MF) and plantain (14,52 Mg of glucose/G MF), Poyo is intermediate (23,03 Mg of glucose/G MF).

Key words: ripening, banana, varieties, physicochemical properties, Musa spp.

\section{Introduction}

Lors du mûrissement de la plupart des fruits climactériques, l'évolution des propriétés organoleptiques est très dépendante du stade de maturité à la récolte (Thompson et Burden, 1995). Elles sont aussi tributaires des conditions de collecte, de transport et de stockage des organes
(Marchal, 1990), ainsi que de la variété (Banks et Joseph, 1991). Généralement, l'observation de la coloration de l'épicarpe et éventuellement celle de la pulpe après une coupure transversale, observées visuellement puis le ramollissement des tissus de l'organe entier apprécié superficiellement par simple touchée permettent aux consommateurs d'établir une relation entre l'état mûr et la qualité du 
fruit (El Zoghbi, 1994 ; Ali et al., 2004 ; Manrique et Lajolo, 2004). Le mûrissement des bananes au plan visuel, se caractérise par le virage progressif de l'épicarpe du vert au jaune selon plusieurs stades dont l'importance contribue à raccourcir ou à prolonger, à une température donnée, la durée totale du processus (Marchal et Nolin, 1990). La coloration de la pulpe traduit la présence ou non de pigments caroténoïdiens précurseurs de la vitamine A (Wall, 2006). Egalement, le ramollissement des fruits est un phénomène crucial qui peut affecter la conservation et la qualité commerciale (Smith et al., 1989 ). Ces critères s'accompagnent d'une amélioration de la saveur qui tient compte de l'équilibre entre les sucres simples obtenus à partir de l'amidon et les acides organiques. Malgré de nombreuses études sur la conservation à long terme (Marcellin, 1974 ; Yao et Dick, 1996, ; Agbo et al., 1996) de la banane, le problème demeure toujours au niveau des pays producteurs. La connaissance des changements physiques et biochimiques liés au mûrissement serait d'un apport important pour la mise en place d'une technologie de conservation appropriée qui retarderait le mûrissement et garderait la qualité du fruit.

La présente étude a été envisagée afin d'évaluer les changements physico-chimiques caractérisant le mûrissement au cours de l'entreposage de trois variétés de bananes Musa spp. (AAB, cv. Corne 1 ; AAA, cv. Poyo et AA, cv. Figue Sucrée).

\section{Matériel et méthodes}

\subsection{Matériel végétal}

L'étude a été faite sur trois variétés de bananes les plus répandus et les plus consommés en Côte d'Ivoire :

- banane à cuire plantain triploïde $A A B$, cultivar Corne 1 ;

- banane dessert triploïde AAA, cultivar Poyo ;

- banane dessert diploïde AA, cultivar Figue Sucrée.

Les échantillons utilisés proviennent des plantations paysannes de M'pouto, village situé dans la banlieue Sud d'Abidjan à une dizaine de kilomètres de l'université de Cocody Abidjan.à $05^{\circ} 19^{\prime} 29,3^{\prime \prime}$ de latitude Nord et $003^{\circ}$ 57'32, 5" de longitude Ouest à une altitude de $8 \mathrm{~m}$. Les fruits ont été récoltés 85 à 95 jours après l'émergence de la hampe florale, au moment du noircissement de l'apex des organes indiquant la maturité physiologique des bananes (Dadzie et Orchard, 1997).

\subsection{Méthodes}

Deux régimes par variétés et par répétition ont été sélectionnés au champ, puis convoyés au laboratoire. Les fruits des deuxièmes et troisièmes mains ont été choisis pour les expériences. Leur masse varie de 300 à $370 \mathrm{~g}$ pour le plantain, 165 à $190 \mathrm{~g}$ pour le Poyo et 44 à $48 \mathrm{~g}$ pour la Figue Sucrée. Après rinçage à l'eau de robinet, les fruits ont été entreposés sur des clayettes ouvertes à température ambiante de $28 \pm 2^{\circ} \mathrm{C}$ et à humidité relative de $80-90 \%$. Au cours du mûrissement, trois fruits sont sélectionnés au hasard par simple observation visuelle de la coloration de l'épicarpe dans chaque lot de variété à chaque fois qu'un stade de mûrissement apparaissait par comparaison avec l'échelle colorimétrique de l'épicarpe choisie pour les mesures et dosages.

\subsubsection{Détermination des stades de mûrissement}

Les stades du mûrissement ont été caractérisés en observant les changements de couleur de l'épicarpe et en les comparant avec une échelle colorimétrique établie par IRFA (1980) pour l'appréciation des stades de mûrissement des bananes commercialement admis. Les stades de coloration vont du vert (V) au jaune tigré (JT) en passant par des stades intermédiaires que sont vert clair (VC) ; tournant vert (TV) ; tournant jaune (TJ) ; jaune aux extrémités vertes (JV) et jaune (J).

\subsubsection{Délai de mûrissement}

Le délai moyen de mûrissement (temps moyen que met chaque variété pour mûrir) et celui de l'apparition des différents stades, ont été estimés en jours depuis la récolte jusqu'à l'apparition successive de chaque coloration.

\subsubsection{Indice colorimétrique de la pulpe}

L'indice colorimétrique de la pulpe pendant le mûrissement, a été déterminé en se référant à un nuancier gradué en unités arbitraires (ua) de 2 à 16 , soit du blanc- crème à l'orangé (IRFA, 1980). 


\subsubsection{Résistance pénétrométrique}

La mesure de la fermeté des fruits entiers a été faite à l'aide d'un pénétromètre du type arbalète. Elle consiste à évaluer en kilogrammes, la résistance à la pénétration de l'embout cylindrique de l'instrument (longueur $=8 \mathrm{~mm}$, diamètre $=3$ $\mathrm{mm}$ ) à l'intérieur du fruit.

\subsubsection{Glucides solubles et réducteurs}

Le dosage des glucides solubles et réducteurs a été fait en utilisant l'acide dinitro-3,5-salycilique (DNS). Le principe est basé sur le fait que les sucres réducteurs (glucoses) provenant de la dégradation des macromolécules d'amidon sont, sous l'effet de la chaleur, transformés en furfural. En présence de composés phénoliques tel que le DNS et en milieu alcalin, le furfural donne une coloration jaune orangé caractéristique dont l'absorbance est mesurée par spectro-photométrie à la longueur d'onde de $540 \mathrm{~nm}$. L'extrait glucidique est obtenu par broyage de $2 \mathrm{~g}$ de pulpe de fruit dans $8 \mathrm{ml}$ d'alcool $80 \%$ et une pincée de sable de fontainebleau. Le tout est ensuite centrifugé à $5000 \mathrm{trs} / \mathrm{min}$ pendant $10 \mathrm{~min}$. Le surnageant est recueilli et le volume est complété à $25 \mathrm{ml}$ avec de l'alcool $80 \%$, puis dosé. Les résultats rapportés en équivalents glucose, sont exprimés en milligrammes par gramme de matière fraîche (mg /g MF).

\subsubsection{Acidité titrable totale}

La méthode utilisée pour la détermination de l'acidité est le titrage de jus de pulpe à l'hydroxyde de sodium $(\mathrm{NaOH} 0,1 \mathrm{~N})$ en présence de phénolphtaléine. Le jus de pulpe est obtenu après broyage de $30 \mathrm{~g}$ de pulpe de banane dans $90 \mathrm{ml}$ d'eau distillée et centrifugé à $5000 \mathrm{trs} / \mathrm{min}$ pendant $10 \mathrm{~min}$. Les résultats sont exprimés en milliéquivalents pour 100 grammes de matière fraîche (méq / 100g MF).

\subsubsection{Analyses statistiques}

Les résultats ont été soumis à l'analyse de variance (ANOVA) avec le logiciel STATISTICA 6.0. En cas de différences significatives, les moyennes sont comparées par le test de Newman-Keuls au seuil de $5 \%$.

\section{Résultats}

\subsection{Stades et délais de mûrissement}

Les résultats indiquent que le temps que met chaque fruit pour passer d'un stade de mûrissement à un autre à travers le déverdissage de l'épicarpe varie en fonction des variétés depuis la récolte jusqu'à la sénescence (Fig.1A). Les tests statistiques montrent que pour les variétés de bananes testées, le délai d'apparition du stade (VC) qui suit immédiatement le stade (V) à la récolte est d'environ 3 jours. Les stades TV ; TJ ; TV et $\mathrm{J}$; TJ s'observent respectivement chez le Poyo entre 5 et 8 jours puis entre 12 et 16 jours après entreposage. Chez le plantain, ces mêmes stades s'observent entre 6 et 11 jours puis 13 et 17 jours et enfin chez la Figue Sucrée entre 4 et 7 jours puis entre 9 et 12 jours après entreposage. Une comparaison entre variétés, indique que les stades $\mathrm{J}$ et JT ont un délai d'apparition statistiquement identique chez le plantain et le Poyo et que ce délai est supérieur à celui de la Figue Sucrée (Fig.1A).

La comparaison du délai du mûrissement de chacune des trois variétés testées montre que le plantain met plus de temps (9 jours) que le Poyo et la Figue Sucrée qui prennent environ 7 jours pour atteindre un stade moyen de mûrissement, dans les conditions de l'étude. Les différences entre les valeurs relevées sont significativement différentes (Fig.1B).

\subsection{Coloration de la pulpe}

Aux stades (V) et (VC), la pulpe est de couleur blanc-crème (indice $=2$ ) chez le Poyo et la Figue Sucrée et jaune clair (indice $=6$ ) chez le plantain (Fig. 2A). Des stades (TV) à (TJ), la couleur de la pulpe du Poyo et de la Figue Sucrée demeure blanc-saumon (indice $=4$ ), alors qu'elle vire progressivement du jaune (indice $=8$ ) au jaune orangé (indice $=10$ ) chez le plantain. Au terme du mûrissement (stade JT), elle est jaune (indice $=8$ ), jaune orangé (indice $=10$ ) et orangé (indice $=14$ ), respectivement chez la Figue Sucrée, le Poyo et le plantain.

Les valeurs moyennes des indices durant tout le mûrissement sont significativement différentes et en relation avec les variétés (Fig. 2B). 

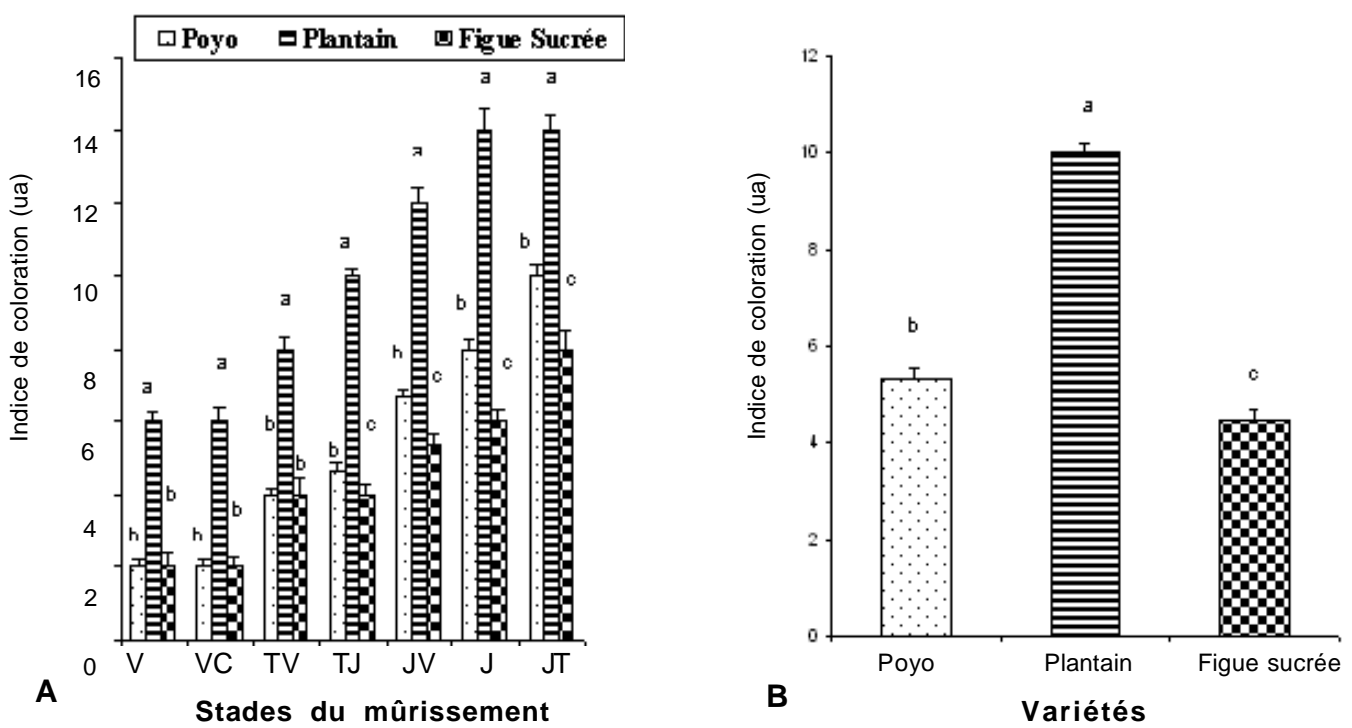

Figure 1 : Délai du mûrissement des variétés de bananes

A : Délai d'apparition des stades du mûrissement

B : comparaison intervariétale des délais moyens du mûrissement au stade JT vert (V) ; clair (VC) ; tournant vert (TV) ; tournant jaune (TJ) ; jaune aux extrémités vertes (JV) ; jaune (J) ; jaune tigré (JT) . $N B$ : Les moyennes suivies par la même lettre minuscule à un stade donné ne sont pas significativement différentes
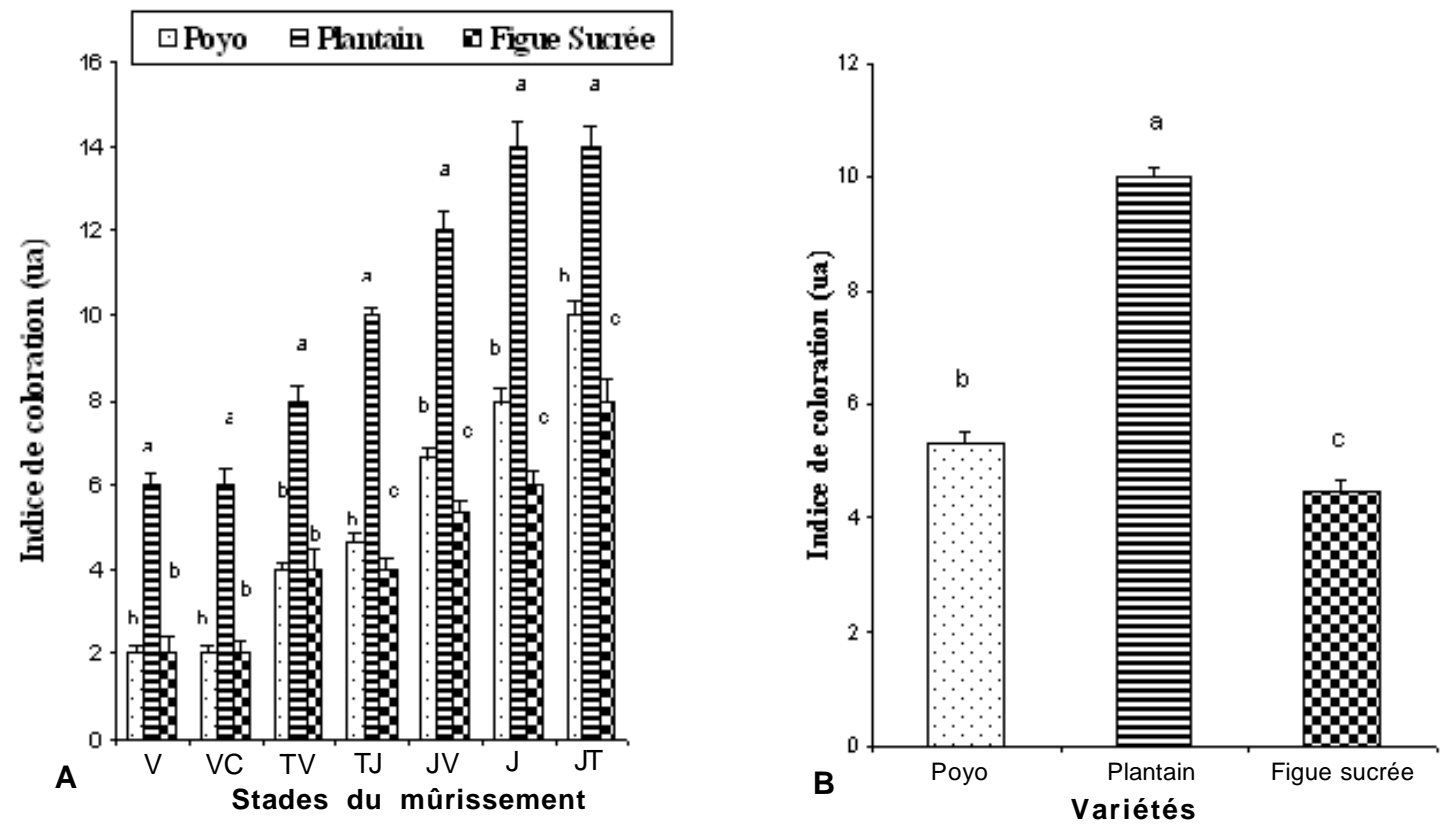

Figure 2 : Coloration de la pulpe des variétés de bananes lors du mûrissement

A : Indice de coloration de la pulpe en fonction du stade du mûrissement

B : comparaison intervariétale des moyennes de l'indice colorimétrique de la pulpe en fin de mûrissement vert (V) ; clair (VC) ; tournant vert (TV) ; tournant jaune (TJ) ; jaune aux extrémités vertes (JV) ; jaune (J) ; jaune tigré (JT)

$N B$ : Les moyennes suivies par la même lettre minuscule à un stade donné ne sont pas significativement différentes 


\subsection{Résistance pénétrométrique}

La fermeté des fruits entiers évaluée sur la base de la résistance pénétrométrique, diminue progressivement pour chaque variété en fonction du stade du mûrissement (Fig. 3A). La baisse de fermeté du plantain, est moins importante que celle du Poyo et de la Figue Sucrée. Ainsi, au stade (V), le poyo et le plantain ont une résistance pénétrométrique statistiquement

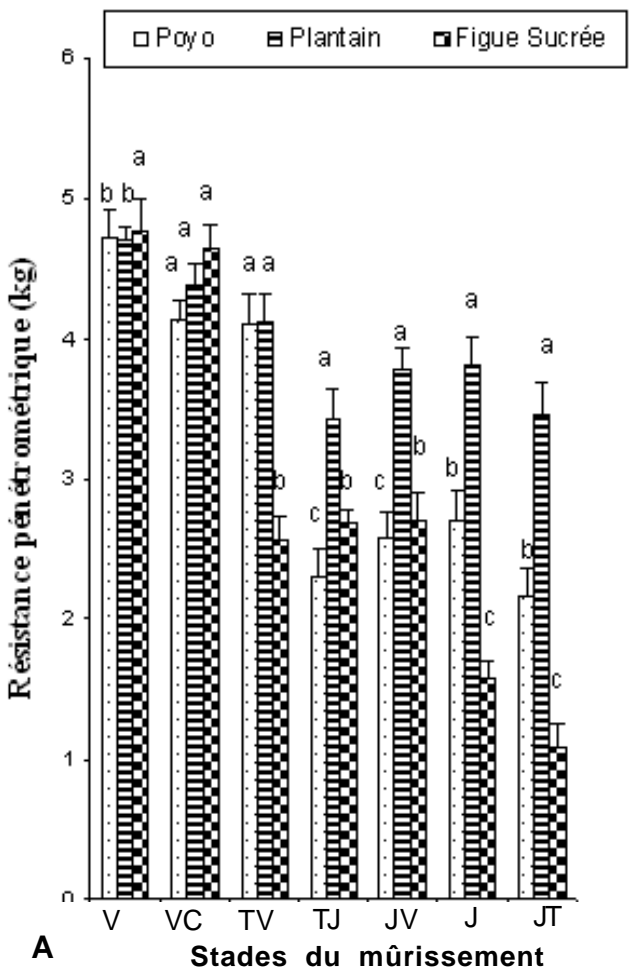

égale $(4,70 \mathrm{~kg})$ et inférieures à celle de la figue sucrée $(4,75 \mathrm{~kg})$. Aux stades (TJ) à (JT), elle varie de 3,45 à $3,50 \mathrm{~kg}$, de 2,30 à 2,15 kg et de 2,65 à $1,10 \mathrm{~kg}$ respectivement chez le plantain, Poyo et Figue Sucrée.

La comparaison intervariétale des résistances pénétrométriques moyennes pendant le mûrissement indique qu'à l'état mûr, le plantain présente une fermeté statistiquement plus élevée que le Poyo et la Figue Sucrée (Fig. 3B).

Figure 3 : Résistance pénétrométrique des variétés de bananes lors du mûrissement

A : Résistance pénétrométrique en fonction du stade du mûrissement

B : comparaison intervariétale des moyennes de la résistance pénétrométrique en fin de mûrissement vert (V) ;clair (VC) ; tournant vert (TV) ; tournant jaune (TJ) ; jaune aux extrémités vertes (JV) ; jaune (J) ; jaune tigré (JT). $N B$ : Les moyennes suivies par la même lettre minuscule à un stade donné ne sont pas significativement différentes

\subsection{Glucides solubles et réducteurs}

L'évolution des taux de sucres solubles et réducteurs dans les fruits (Fig.4A) se déroule en deux phases. La première phase est caractérisée par une intense accumulation de sucres jusqu'à l'obtention d'un optimum suivie de la deuxième phase caractérisée par une baisse.
Les résultats obtenus montrent que l'évolution de ce taux dépend de la variété et du stade du mûrissement. Ainsi, chez le plantain, l'optimum (33,50 mg de glucose / g. MF) est atteint au stade (JV), contrairement au Poyo et la Figue Sucrée chez lesquels les maxima respectifs $(84,26$ et $63,27 \mathrm{mg}$ de glucose / g. MF) sont enregistrés au stade (TJ). 
La comparaison intervariétale des teneurs moyennes en sucres solubles et réducteurs (Fig. 4B) met en évidence des valeurs significativement différentes.
Les variétés accumulant le plus et le moins de sucres solubles et réducteurs sont respectivement la Figue Sucrée (33,80 mg de glucose / g. MF) et le plantain (14,52 mg de glucose / g. MF).
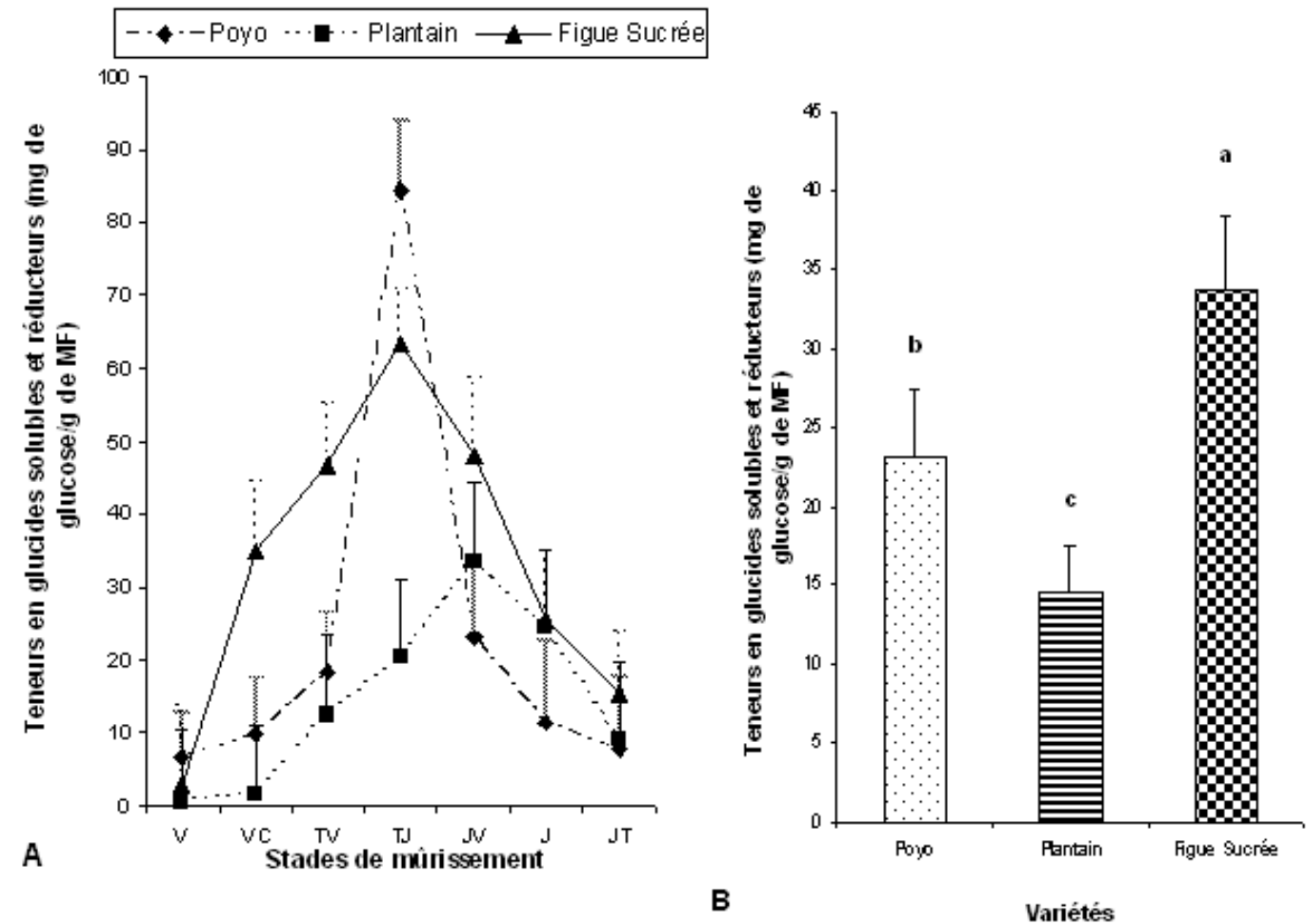

Figure 4 : Glucides solubles et réducteurs dans la pulpe des variétés de bananes lors du mûrissement

A : Teneurs en glucides solubles et réducteurs en fonction du stade du mûrissement

B : comparaison intervariétale des teneurs en glucides solubles et réducteurs moyennes de la résistance pénétrométrique en fin de mûrissement

vert (V) ; clair (VC) ; tournant vert (TV) ; tournant jaune (TJ) ; jaune aux extrémités vertes (JV) ; jaune (J) ; jaune tigré (JT).

$N B$ : Les moyennes suivies par la même lettre minuscule à un stade donné ne sont pas significativement différentes

\subsection{Acidité titrable totale}

Quelle que soit la variété, l'acidité titrable totale augmente jusqu'à atteindre un pic qualifié de "stade de plein mûrissement» caractéristique de chaque variété avant de baisser (Fig. 5A). Le pic de l'acidité titrable est atteint au stade (JV) chez le plantain et le poyo quand à la figue sucrée, c'est au stade suivant (J) qu'il est observé. Les valeurs moyennes de l'acidité titrable totale sont statistiquement identiques chez le Poyo et le plantain (environ 14,28 méq / 100g. MF) et plus élevées chez la Figue Sucrée $(22,17$ méq / 100g. MF) pendant tout le mûrissement (Fig. 5B). 

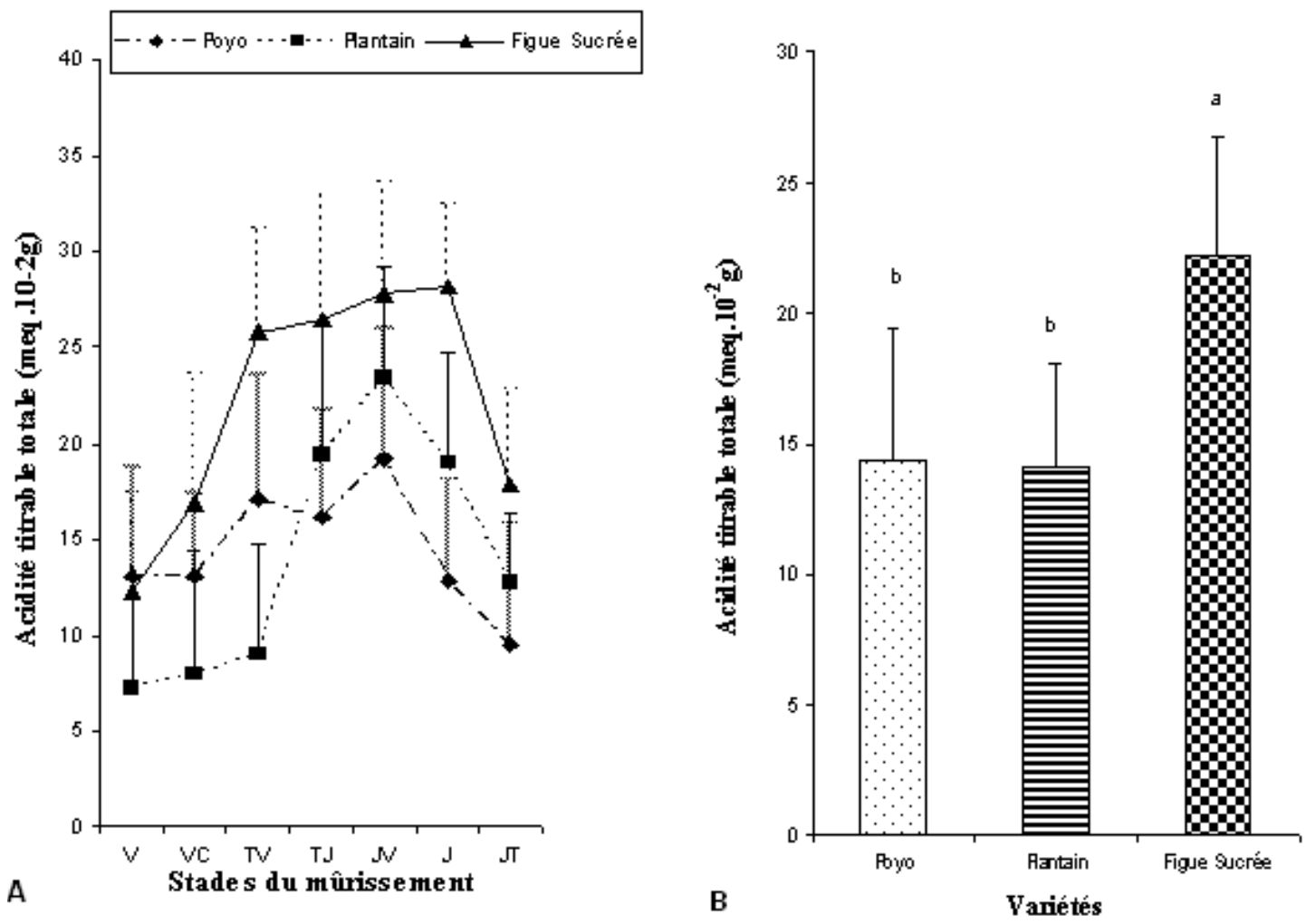

Figure 5 : Acidité titrable totale de la pulpe des variétés de bananes lors du mûrissement A : Acidité titrable totale en fonction du stade du mûrissement

B : comparaison intervariétale de l'acidité titrable totale moyenne en fin de mûrissement

vert (V) ; clair (VC) ; tournant vert (TV) ; tournant jaune (TJ) ; jaune aux extrémités vertes (JV) ; jaune (J) ; jaune tigré (JT) . $N B$ : Les moyennes suivies par la même lettre minuscule à un stade donné ne sont pas significativement différentes

\section{Discussion}

Le mûrissement des fruits de trois variétés de bananes étudiées au cours de l'entreposage à température ambiante $\left(28 \pm 2^{\circ} \mathrm{C}\right)$ et à humidité relative de $80-90 \%$ se déroule par étapes successives comme l'a montré Von Loesseck (1950). Cela se traduit extérieurement par le virage progressif de la coloration de l'épicarpe, du vert au jaune tigré en passant par des étapes intermédiaires allant du vert clair au jaune. Cette évolution serait due à la dégradation des chlorophylles par un ensemble d'oxydases dont ferait partie la chlorophylle oxydase, liée à la membrane thylakoïdale des chloroplastes (Matile, 1980 ; Martinoia et al., 1982 ; Blackbourn et al.,1989 ). La destruction des pigments chlorophylliens aurait pour effet de laisser apparaître les caroténoïdes initialement présents dans les organes verts. Relativement à la dépigmentation chlorophyllienne de l'épicarpe suivie du jaunissement, la Figue Sucrée achève son mûrissement avant celui du Poyo et du plantain. Cette observation pourrait être liée à l'optimum thermique du mûrissement et à la consistance de l'épicarpe qui seraient différents selon les variétés.

Au terme du mûrissement, la pulpe initialement blanche vire à l'orangé, au jaune orangé ou au jaune clair respectivement chez le plantain, le Poyo et la Figue Sucrée. Ces différentes colorations observées à la fin du mûrissement semblent 
dépendre de la richesse en carotènes, précurseurs de la vitamine A de chacune des variétés (Wall, 2006). Ainsi, la pulpe du plantain qui est la plus orangée serait celle qui serait la plus riche en vitamine $A$ comparativement aux bananes dessert que sont le Poyo et la Figue Sucrée (Chandler, 1995).

Le faible ramollissement chez le plantain par rapport au Poyo et à la Figue Sucrée semble être spécifique à cette variété. Ce phénomène parait tributaire, comme le supposent Thompson et Burden (1995), de trois évènements que sont la transformation de l'amidon (élément de structure) en sucres simples, la dégradation des parois cellulaires par les cellulases et une migration osmotique de l'eau du péricarpe vers la pulpe. En effet, ces réactions sont moins rapides chez le plantain que chez les deux autres variétés testées (John et Marchal, 1995) qui possèdent de surcroît un péricarpe beaucoup moins épais.

L'augmentation de la teneur en sucres solubles et réducteurs au niveau de toutes les variétés de banane, pourrait s'expliquer par une dégradation de l'amidon contenu dans leur pulpe respective. Ainsi, au cours du mûrissement, l'amidon de la Figue Sucrée et celui du Poyo sont plus hydrolysés par les enzymes telles que l'áamylase que chez la banane plantain. Des résultats similaires ont été obtenus par Marchal et al., (1988) sur Giant Cavendish (banane dessert) et par Collin et Dalnic (1991) sur le plantain Orishele. De même, Mariott et al., (1991) ont révélé l'existence d'une faible hydrolyse de l'amidon chez la banane plantain que chez la banane dessert durant tous les stades de mûrissement. L'amidon est donc complètement ou suffisamment dégradé chez les bananes de dessert contrairement à la banane plantain d'où des pics plus élevés de teneurs en glucides chez ces premières.

L'évolution de l'acidité titrable au cours du mûrissement des bananes testées, sont globalement identiques à celles observées par Marchal et al. (1988) et par Collin et Dalnic (1991) qui ont montré que l'acidité titrable des bananes dessert augmente jusqu'au stade JV avant de baisser. Au cours du mûrissement, l'augmentation des teneurs en acides dans la pulpe serait le fait des acides organiques, en particulier les acides citriques, maliques et oxaloacétiques (Satyan et Patwardhan, 1984 ;
Inaba et Nakamura, 1988). Selon John et Marchal (1995), la baisse des teneurs en acides observée après que le pic ait été atteint, serait due à l'utilisation de ces derniers comme substrats par de nombreuses enzymes dont la malate synthase, l'enzyme malique et la phosphoenol pyruvate carboxylase lors des activités métaboliques, notamment dans les voies respiratoires.

\section{Conclusion}

Les analyses effectuées sur les bananes fraîchement récoltées jusqu'au mûrissement total, ont montré que le délai d'apparition des stades du mûrissement sont fonction des variétés. Le plantain mûrit plus lentement que les bananes dessert. En outre, l'indice de coloration, la résistance pénétrométrique, la teneur en glucides solubles et réducteurs ainsi que l'acidité titrable varient en fonction du stade de mûrissement et des variétés. Cependant, l'évolution de ces paramètres marquant le mûrissement est très caractéristique de chaque variété: A l'état mûr, le plantain présente une couleur jaune orangé de la pulpe quand le Poyo indique une couleur jaune clair et la Figue Sucrée blanc-saumon. La résistance pénétrométrique est plus élevée chez le plantain que chez les bananes de dessert. Les variétés accumulant le plus et le moins de sucres solubles et réducteurs sont la Figue Sucrée et le plantain, le Poyo ayant un caractère intermédiaire. L'acidité titrable est élevée au niveau de la banane plantain et du Poyo contrairement à la Figue Sucrée.

\section{Références citées}

Ali Z.M., Chin L. \& Lazan H., 2004. A comparative study on wall degrading enzymes, pectin modifications and softening during ripening of selected tropical fruits. PI. Sci., 167: 317-327.

Agbo N.G., Soumanou M. and Yao K. A, 1996. Nouvelles techniques de conservation de la banana plantain en milieu rural avec de la matière végétale locale. Sciences des aliments, 16 : 607-621.

Banks N.H. \& Joseph M., 1991. Factors affecting resistance of banana fruit to compression and impact bruising. J. Sci. Food Agric. 56: 315-323. 
Blackbourn H.D., John P. \& Jeger M.J., 1989. Ultrastructural and biochemical changes accompanying degreening in bananas and plantains at tropical temperatures. Aspects Appl. Biol. 20: 83-84.

Chandler S. 1995. The nutritional value of bananas. In: Gowen S., ed. Bananas and Plantains. Chapman \& Hall. London. pp. 468-480.

Collin M.N. \& Dalnic R., 1991. Evolution de quelques critères physico-chimiques de la banane plantain (cv Orishelé) au cours de la maturation. Fruits. 46: 13-17.

Dadzie B.K. \& Orchard J.E., 1997. Evaluation postrécolte des hybrides de bananiers et bananiers plantain : critères et méthodes .Guides techniques INIBAP2. IPGRI / INIBAP / CTA (Rome, Montpellier \& Wageningen). 63 pp.

El Zoghbi M., 1994. Biochemical changes in some tropical fruits during ripening. Food Chemistry. 49: 33-37.

Inaba A. \& Nakamura R., 1988. Numerical expression for estimating the minimum ethylene exposure time necessary to induce ripening in banana fruit. J. Am. Soc. Hortic. Sci. 113 (4): 561-564

IRFA, 1980 : Institut de Recherches sur les Fruits et Agrumes. La qualité de la banane. La Réglementation française et son Interprétation. 74 pp.

John P. \& Marchal J., 1995. Ripening and biochemistry of fruit. In: Gowen S., ed. Bananas and Plantains. Chapman \& Hall. London. pp. 434-467.

Manrique G.D. \& Lajolo L.M., 2004. Cell-wall polysaccharide modifications during post harvest ripening of papaya fruit (Carica papaya). Post harv. Biol. Technol. 33: 11-26.

Marcellin P., 1974. Conservation de fruits et légumes en atmosphère contrôlée à l'aide de membranes de polymères. Revue gén. Froid, $3: 217-225$.

Marchal J., 1990. Contraintes post-récoltes et perspectives d'amélioration de la manipulation du stockage et de la transformation du plantain et des autres bananes à cuire en Afrique de l'Ouest. Fruits. 45 (5): 439-445.

Marchal J. \& Nolin J., 1990. Bananes. Qualité des fruits, Physiologie avant et après récolte. Fruits. Spécial Bananes. 119-122.

Marchal J., Nolin J., \& Letory J., 1988. Influence sur la maturation de l'enrobage de bananes avec Semper-fresh. Fruits. 43: 447-453.

Mariott J., Robinson M. \& Karikari S.K., 1981. Starch and sugar transformation during ripening of plantains and bananas. J. Sci. Food. Agric. 32: 1021-1026.

Martinoia E., Dalling M.J. \& Matile P., 1982. Catabolism of chlorophyll, demonstration of chloroplast localised peroxydative and oxydative activities. Z. Pflanzenphysiol. 109: 269-279.

Matile P., 1980. Catabolism of chlorophyll: involvement of peroxydase. Z. Pflanzenphysiol. 99: 475-478.

Satyan S.H. \& Patwardhan M.V., 1984. Purification and regulatory properties of phosphoenolpyruvate carboxylase from banana fruits of Dwarf Cavendish. J. Food Sci. Technol. 21: 135-138.

Smith S.J.S., Tucker G.A. \& Jeger M.J., 1989. Softening and cell wall changes in bananas and plantain. Aspects Appl. Biol. 20: 57-65.

Thompson A.K. \& Burden O.J., 1995. Harvesting and fruit care. In: Gowen S., ed. Bananas and Plantains. Chapman \& Hall. London. pp. 403-433.

Von Loesecke, 1950. Chemical changes during ripening bananas. Chem. Physiol. Technol. 4 $67-118$.

Wall M.M., 2006. Ascorbic acid, vitamin A and mineral composition of banana (Musa sp.) and papaya (Carica papaya) cultivars grown in Hawaii. J Food Compo Anal. 19: 434-445.

Yao K.A. et Dick E., 1996. Conservation du plantain en atmosphère contrôlée à l'aide d'une membrane silicone. Agron. Afr. VIII (3) : 193-199. 